\title{
Texture Dirac Mass Matrices and Lepton Asymmetry in the Minimal Seesaw Model with Tri-Bimaximal Mixing
}

\author{
Agus Purwanto, ${ }^{1, *}$ Bintoro Anang Subagyo, ${ }^{1, \dagger}$ and Erika Rani ${ }^{2, \ddagger}$ \\ ${ }^{1}$ Jurusan Fisika-FMIPA, Institut Teknologi Sepuluh Nopember \\ Kampus ITS Sukolilo, Surabaya 61111 \\ ${ }^{2}$ Jurusan Fisika, UIN Maulana Malik Ibrahim, Jl. Gajayana 50, Malang 65114
}

\begin{abstract}
We examined the minimal seesaw mechanism of $3 \times 2$ Dirac matrix by starting our analysis with the masses of light neutrinos with tri/bi-maximal mixing in the basis where the charged-lepton Yukawa matrix and heavy Majorana neutrino mass matrix are diagonal. We found all possible Dirac mass textures which contain one zero entry or two in the matrix.
\end{abstract}

KEYWORDS: tri-bimaximal mixing, minimal seesaw model, mass matrices and lepton asymmetry

\section{INTRODUCTION}

The recent neutrino oscillation experiments have provided us with robust evidence that neutrinos have tiny masses and their flavor mixing involves two large angles and one small angle [x]. A global analysis of current neutrino oscillation data yields $7.2 \times 10^{-5} V^{2} \leq \Delta m_{\text {sol }}^{2} \leq 8.9 \times 10^{-5} V^{2}$ and $1.7 \times 10^{-3} V^{2} \leq \Delta m_{a t m}^{2} \stackrel{s}{\leq} 3.3 \times 10^{-3} V^{2}$ for the squared mass differences of solar and atmospheric neutrinos and $30^{\circ} \leq \theta_{12} \leq 38^{\circ}, 36^{\circ} \leq \theta_{23} \leq 54^{\circ}$ and $0^{\circ} \leq \theta_{13} \leq 10^{\circ}$ for the flavor mixing angles at the $99 \%$ confidence level (the best-fit values are $\Delta m_{\text {sol }}^{2}=8.0 \times 10^{-5} V^{2}$, $\Delta m_{a t m}^{2}=2.5 \times 10^{-3} V^{2}, \theta_{12}=34^{\circ}, \theta_{23}=45^{\circ}$ and $\theta_{13}=0^{\circ}[\mathrm{y}]$. Where we define $\Delta m_{\text {sol }}^{2}=m_{2}^{2}-m_{1}^{2}$ and $\Delta m_{\text {atm }}^{2}=\left|m_{3}^{2}-m_{2}^{2}\right|$ with the neutrino mass eigenvalues $m_{1}, m_{2}$ and $m_{3}$. The on-going and forthcoming neutrino oscillation experiments will shed light on the sign of $\Delta_{a t m}$ and the magnitude of $\theta_{13}$ and even the CP-violating phase.

The seesaw mechanism is arguably the most attractive way to explain the smallnes of neutrino masses. In its conventional form, the seesaw invokes three heavy singlet right-handed neutrinos $\nu_{R}$ [1]. However, the number three is not sacret. The two non-zero light neutrino mass difference required by experiment could be explained with just two heavy right-handed neutrinos [2]. The seesaw mechanism with two right-handed neutrinos predicts one of the physical light neutrino mass to be exactly zero; which is permissible within the current knowledge of neutrino masses and mixings.

On the other hand, the observed neutrino mixing matrix is compatible with the so called tri-bimaximal form, introduced by Harrison, Perkins and Scott [3]. This tri-bimaximal

\footnotetext{
*E-MAIL: purwanto@physics.its.ac.id

†E-MAIL: b_anang@physics.its.ac.id

¥E-MAIL: erikarani@gmail.com
}

neutrino mixing is based on the idea that there are both bi-maximal $(0,1,1) / \sqrt{2}$ as well as tri-maximal $(1,1,1) / \sqrt{3}$ mixings in the lepton sector.

The results of the LEP experiments on the measurement of the invisible width of the $\mathrm{Z}$ boson imply that only three flavor neutrinos exist in nature (see Ref.[4]). The simplest one to give $3 \times 3$ mass matrix $m_{\nu}$ is both Dirac mass matrix $m_{D}$ and right-handed massive Majorana mass matrix $M_{R}$ are $3 \times 3$ matrices.

\section{THE MINIMAL SEESAW MODEL}

The most economical seesaw model which compatible with solar and atmospheric neutrinos is satisfied by two righthanded neutrinos. The leptonic part of the Yukawa interactions in presence of three left-handed and two right-handed neutrinos can be written as

$$
\begin{aligned}
-\mathcal{L}= & \overline{\psi_{L i}}\left(Y_{\nu}\right)_{i j} \tilde{\phi} N_{R j}+\overline{\psi_{L i}}\left(Y_{\ell}\right)_{i j} \phi e_{R j} \\
& +\frac{1}{2} \overline{N_{R i}^{c}}\left(M_{R}\right)_{i j} N_{R j}+\text { h.c. }
\end{aligned}
$$

where $N_{R}$ denote the right-handed neutrino fields which are singlet under the standard model gauge group, $\phi$ is $\mathrm{SU}(2)$ higgs doublet with $\tilde{\phi}=i \sigma_{2} \phi^{*}, \psi_{L i}$ is the lepton doublet of flavor i, and $E_{R i}$ are the right-handed charged leton singlet. the Yukawa coupling constants $Y_{\nu}$ and $Y_{\ell}$ are comlex-valued matrices. After the electroweak symmetry breaking one gets the charged mass matrix $m_{\ell}=v Y_{\ell}$ and the Dirac mass matrix for the neutrino as $m_{D}=v Y_{\nu}$ where $v$ is the vacuum exectation value of the neutral component of the higgs doublet $\phi$. The Majorana mass matrix $M_{R}$ is $2 \times 2$ comlex symmetric matrix. The mass matrix for the neutral fermions can be written as

$$
M_{\nu}=\left(\begin{array}{cc}
0 & m_{D} \\
m_{D}^{T} & M_{R}
\end{array}\right)
$$


The light neutrino mass matrix $m_{\nu}$ after the seesaw diagonalization is given by the seesaw formula

$$
m_{\nu}=-m_{D} M_{R}^{-1} m_{D}^{T}
$$

This master formula (3) is valid when the eigenvalues of $M_{R}$ are much larger than the elements of $m_{D}$ and in such a case the eigenvalues of $m_{\nu}$ come out very small with respect to those of $m_{D}$.

In general the Majorana mass matrix $M_{R}$ is non-diagonal form in the basis where the charged current is flavor diagonal. In this form one can make a basis rotation so that the righthanded Majorana mass matrix becomes diagonal by the unitary matrix. All possible heavy Majorana mass matrices $M_{R}$, their inverse and their diagonal form are given in the Table I, with the values

$$
d_{ \pm}=\frac{M_{2} \pm \sqrt{4 M_{1}^{2}+M_{2}^{2}}}{2}
$$

and

$$
D_{ \pm}=\frac{M_{1}+M_{3} \pm \sqrt{M_{1}^{2}+4 M_{2}^{2}-2 M_{1} M_{3}+M_{3}^{2}}}{2}
$$

\section{TABLE I: The Heavy Majorana Mass Matrices}

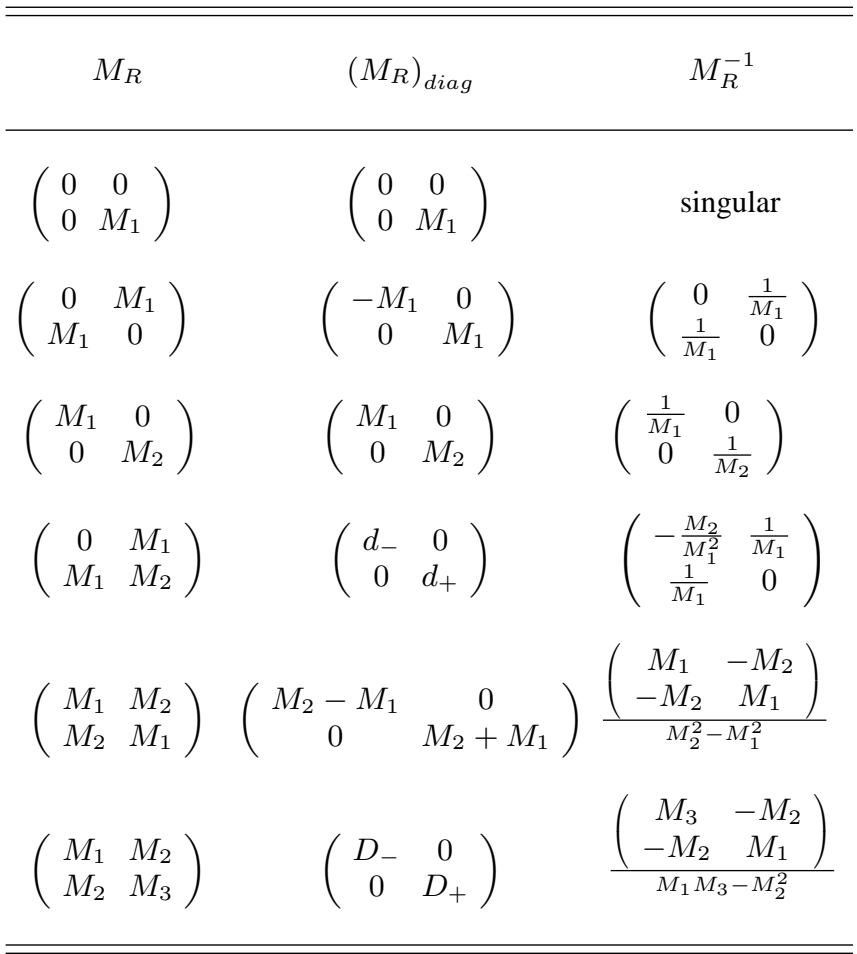

In this form the Dirac mass matrix must be $3 \times 2$ form. It implies the the determinant of light massive neutrino matrix is zero

Since

$$
\operatorname{det} m_{\nu}=0
$$

$$
U\left(\begin{array}{lll}
a_{1} & a_{2} & a_{3} \\
b_{1} & b_{2} & b_{3} \\
c_{1} & c_{2} & c_{3}
\end{array}\right) V=\left(\begin{array}{ccc}
d_{1} & 0 & 0 \\
0 & d_{2} & 0 \\
0 & 0 & d_{3}
\end{array}\right)
$$

it gives at least one of the eigenvalues of $m_{\nu}$ is exactly zero; i.e, either $m_{1}=0$ for normal neutrino mass hierarchy ( $\left.m_{1}<m_{2}<m_{3}\right)$ or $m_{3}=0$ for inverted neutrino mass hierarchy $\left(m_{1}>m_{2}>m_{3}\right)$.

\section{TRI-BIMAXIMAL MIXING AND DIRAC MASS TEXTURE}

It is an experimental fact that within measurement errors the observed neutrino mixing matrix is compatible with the so called tri-bimaximal form, introduced by Harrison, Perkins and Scott (HPS). The matrix is given by

$$
V=\left(\begin{array}{ccc}
\sqrt{\frac{2}{3}} & \frac{1}{\sqrt{3}} & 0 \\
-\frac{1}{\sqrt{6}} & \frac{1}{\sqrt{3}} & \frac{1}{\sqrt{2}} \\
-\frac{1}{\sqrt{6}} & \frac{1}{\sqrt{3}} & -\frac{1}{\sqrt{2}}
\end{array}\right)
$$

One purpose of this paper is just to find the Dirac neutrino mass matrix for either $\mathrm{m} 1=0$ or $\mathrm{m} 3=0$. Looking back to Eqs. (1), we have $3 \times 2$ matrix $m_{D}=v Y_{\nu}$, we can write it in the form

$$
m_{D}=\left(\begin{array}{ll}
x_{1} & y_{1} \\
x_{2} & y_{2} \\
x_{3} & y_{3}
\end{array}\right)
$$

Using the diagonal inverse matrix $M_{R}^{-1}$ we obtain

$$
\begin{aligned}
m_{\nu} & =\left(\begin{array}{ll}
x_{1} & y_{1} \\
x_{2} & y_{2} \\
x_{3} & y_{3}
\end{array}\right)\left(\begin{array}{cc}
\frac{1}{M_{1}} & 0 \\
0 & \frac{1}{M_{2}}
\end{array}\right)\left(\begin{array}{ccc}
x_{1} & x_{2} & x_{3} \\
y_{1} & y_{2} & y_{3}
\end{array}\right) \\
& =\left(\begin{array}{ccc}
\frac{x_{1}^{2}}{M_{1}}+\frac{y_{1}^{2}}{M_{2}} & \frac{x_{1} x_{2}}{M_{1}}+\frac{y_{1} y_{2}}{M_{2}} & \frac{x_{1} x_{3}}{M_{1}}+\frac{y_{1} y_{3}}{M_{2}} \\
\frac{x_{1} x_{2}}{M_{1}}+\frac{y_{1} y_{2}}{M_{2}} & \frac{x_{2}^{2}}{M_{1}}+\frac{y_{2}^{2}}{M_{2}} & \frac{x_{2} x_{3}}{M_{1}}+\frac{y_{2} y_{3}}{M_{2}} \\
\frac{x_{1} x_{3}}{M_{1}}+\frac{y_{1} y_{3}}{M_{2}} & \frac{x_{2} x_{3}}{M_{1}}+\frac{y_{2} y_{3}}{M_{2}} & \frac{x_{3}}{M_{1}}+\frac{y_{3}^{2}}{M_{2}}
\end{array}\right)(10)
\end{aligned}
$$

Then applying the HPS matrix to diagonalize the neutrino mass matrix $m_{\nu}$

$$
V^{T} m_{\nu} V=\left(\begin{array}{l}
m_{i j} \\
\end{array}\right)
$$

we have 


$$
\begin{aligned}
& m_{11}=\frac{\left(4 y_{1}^{2}+y_{2}^{2}+y_{3}^{2}-4 y_{1}\left(y_{2}+y_{3}\right)+2 y_{2} y_{3}\right) M_{1}+\left(4 x_{1}^{2}+x_{2}^{2}+x_{3}^{2}-4 x_{1}\left(x_{2}+x_{3}\right)+2 x_{2} x_{3}\right) M_{2}}{6 M_{1} M_{2}} \\
& m_{12}=\frac{\left(2 y_{1}^{2}-y_{2}^{2}-y_{3}^{2}+y_{1}\left(y_{2}+y_{3}\right)-2 y_{2} y_{3}\right) M_{1}+\left(2 x_{1}^{2}-x_{2}^{2}-x_{3}^{2}+x_{1}\left(x_{2}+x_{3}\right)-2 x_{2} x_{3}\right) M_{2}}{3 \sqrt{2} M_{1} M_{2}} \\
& m_{13}=\frac{\left(2 y_{1}-y_{2}-y_{3}\right)\left(y_{2}-y_{3}\right) M_{1}+\left(2 x_{1}-x_{2}-x_{3}\right)\left(x_{2}-x_{3}\right) M_{2}}{2 \sqrt{3} M_{1} M_{2}} \\
& m_{23}=\frac{\left(y_{1}+y_{2}+y_{3}\right)\left(y_{2}-y_{3}\right) M_{1}+\left(x_{1}+x_{2}+x_{3}\right)\left(x_{2}-x_{3}\right) M_{2}}{\sqrt{6} M_{1} M_{2}} \\
& m_{22}=\frac{\left(y_{1}+y_{2}+y_{3}\right)^{2} M_{1}+\left(x_{1}+x_{2}+x_{3}\right)^{2} M_{2}}{3 M_{1} M_{2}} \\
& m_{33}=\frac{\left(y_{2}-y_{3}\right)^{2} M_{1}+\left(x_{2}-x_{3}\right)^{2} M_{2}}{2 M_{1} M_{2}}
\end{aligned}
$$$$
V^{T} m_{\nu} V \equiv \hat{m}_{\nu}=\left(d_{i} \delta_{i j}\right)
$$

(inverted neutrino mass hierarchy, IH) has no conflict with the present neutrino oscillation measurements. In both cases, the non-vanishing neutrino masses can be determined in terms of $m_{\text {sol }}^{2}$ anad $m_{a t m}^{2}$ :

The lightest neutrino is allowed to be massless; i.e., either $m_{1}=0$ (normal neutrino mass hierarchy, $\mathrm{NH}$ ) or $m_{3}=0$

$$
\begin{gathered}
m_{1}=0 \Rightarrow\left\{\begin{array}{c}
m_{2}=\sqrt{m_{\text {sol }}^{2}} \approx 8.97 \times 10^{-3} \mathrm{eV}, \\
m_{3}=\sqrt{m_{\text {atm }}^{2}+m_{\text {sol }}^{2}} \approx 5.08 \times 10^{-2} \mathrm{eV} .
\end{array}\right. \\
m_{3}=0 \Rightarrow\left\{\begin{array}{c}
m_{1}=\sqrt{m_{\text {atm }}^{2}-m_{\text {sol }}^{2}} \approx 4.92 \times 10^{-2} \mathrm{eV}, \\
m_{2}=\sqrt{m_{\text {atm }}^{2}} \approx 5.00 \times 10^{-2} \mathrm{eV} .
\end{array}\right.
\end{gathered}
$$
chy

These light neutrinos give heavy masses; for normal hierar-

$$
\begin{aligned}
& M_{1}=8.98 \times 10^{12} \mathrm{GeV} \approx 10^{13} \mathrm{GeV} \\
& M_{2}=1.07 \times 10^{14} \mathrm{GeV} \approx 10^{14} \mathrm{GeV}
\end{aligned}
$$

for normal hierarchy, and

$$
\begin{aligned}
& M_{1}=9.12 \times 10^{12} \mathrm{GeV} \approx 10^{13} \mathrm{GeV} \\
& M_{2}=9.07 \times 10^{13} \mathrm{GeV} \approx 10^{14} \mathrm{GeV}
\end{aligned}
$$

for inverse hierarchy.

\section{LEPTON ASYMMETRY}

When the Majorana right-handed neutrinos decay into leptons and Higgs scalars, they violate the lepton number since right-handed neutrino fermionic lines do not have any preferred arrow

$$
\begin{aligned}
& N_{R} \rightarrow \ell+H^{*} \\
& N_{R} \rightarrow \bar{\ell}+H
\end{aligned}
$$

The interference between the tree-level decay amplitude and the absorptive part of the one-loop vertex leads to a lepton asymmetry

$$
\epsilon=\sum_{i=1,2} \frac{\Gamma\left(N_{i} \rightarrow \ell H^{*}\right)-\Gamma\left(N_{i} \rightarrow \bar{\ell} H\right)}{\Gamma\left(N_{i} \rightarrow \ell H^{*}\right)+\Gamma\left(N_{i} \rightarrow \bar{\ell} H\right)}
$$

We assume a hierarchical mass pattern of the heavy neutrinos $M_{1}<<M_{2}$. in this case, the interactions of $N_{1}$ can be in thermal equilibrium when $N_{2}$ decay is washed-out by the lepton number violating processes with ${ }_{1}$. Thus only the decays of $N_{1}$ are relevant for generation of the final lepton asymmetry $\epsilon \approx \epsilon_{1}$. in this case, the CP asymmetry paarameter in the 
TABLE II: Texture Dirac mass matrices and their light neutrino mass matrices

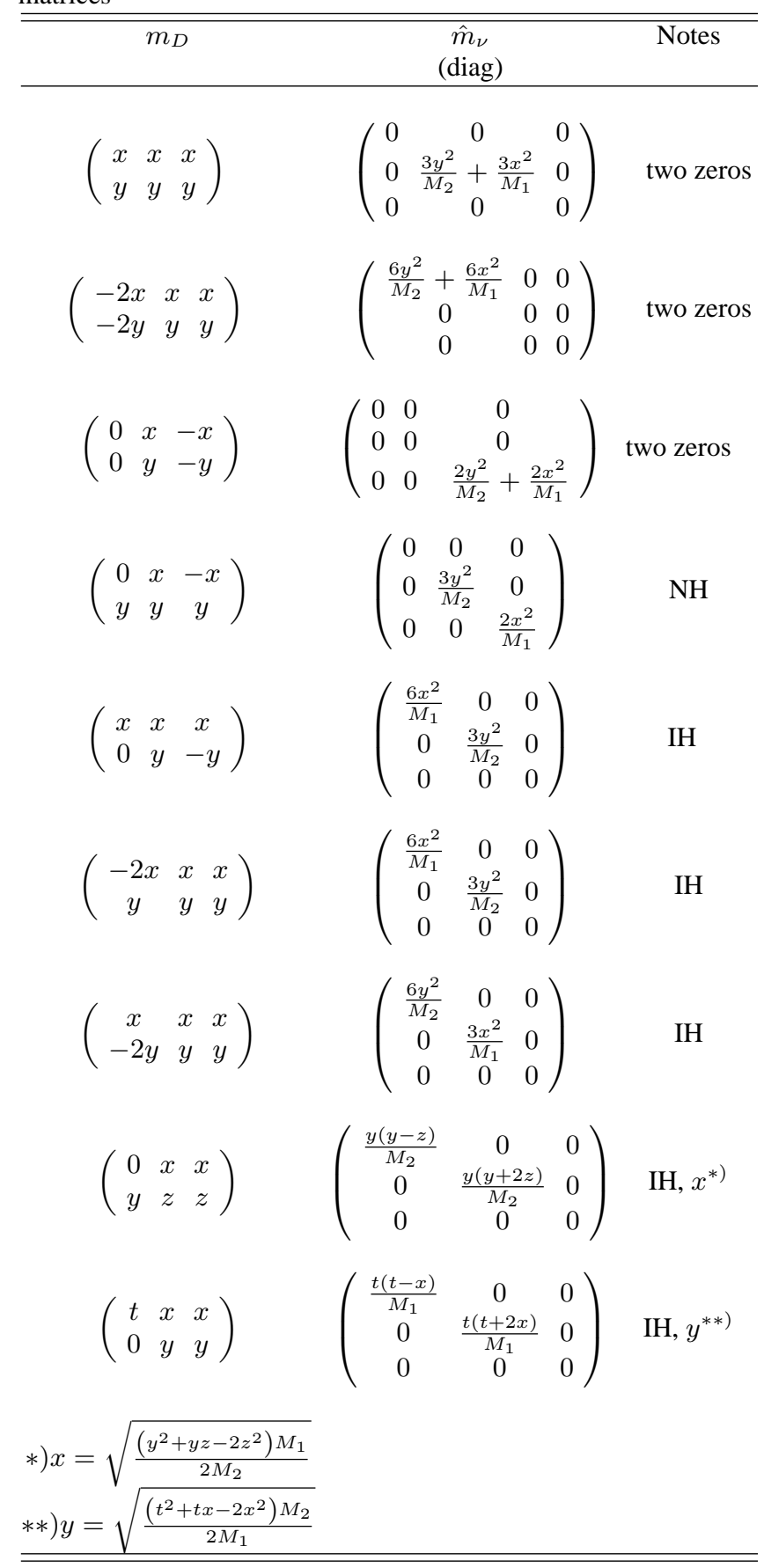

minimal seesaw model is given by

$$
\epsilon=\frac{1}{8 \pi v^{2}} \frac{\Im\left[\left(m_{D}^{\dagger} m_{D}\right)_{12}^{2}\right]}{\left(m_{D}^{\dagger} m_{D}\right)_{11}} f\left(\frac{M_{2}^{2}}{M_{1}^{2}}\right)
$$

where

$$
f(x)=\sqrt{x}\left[1-(1+x) \ln \left(\frac{1+x}{x}\right)+\frac{1}{1-x}\right]
$$

This asymmetry is valid in the basis where the right handed neutrino is diagonal. $f(x) \approx 3 / \sqrt{x}$ for $x>>1$. Sphaleron processes will convert this lepton asymmetry into baryon asymmetry, as a result, the baryon asymmetry is approximately described as

$$
\eta_{B}=0.96 \times 10^{-2}(-\epsilon) \kappa
$$

where $\kappa$ is the efficiency factor, that parameterizes dilution effects for generated lepton asymmetry through washing-out processes.

\section{CONCLUSION}

In this paper we analyze the texture zeros in the neutrino Yukawa Coupling matrix $m_{D}$ and the heavy Majorana neutrino mass matrix $M_{R}$ in the context of the minimal seesaw model including 2 heavy right-handed neutrinos. We illustrate which textures are compatible with the present neutrino oscillation data and discuss their implications for the future neutrino experiments. We do not make the assumption that $M_{R}$ is diagonal. We examined the minimal seesaw mechanism of $3 \times 2$ Dirac matrix by starting our analysis with the masses of light neutrinos with tri/bi-maximal mixing in the basis where the charged-lepton Yukawa matrix and heavy Majorana neutrino mass matrix are diagonal. We found all possible Dirac mass textures which contain one zero entry or two in the matrix.

\section{Acknowledgments}

The authors thank Indonesia Center for Theoretical and Mathematical Physics (ICTMP) for fruitful seminar and discussions.
[1] T. Yanagida, in Proceedings of the Workshop on Unified Theories and Baryon Number of the Universe (Editors: O.Sawada and A. Sugamoto, Tsukuba, Japan, 1979) KEK Report KEK-7918, page 95. M. Gell-Mann, P. Ramond and R. Slansky, in Supergravity (Editors: D.Z. Freedman and P. Van Nieuwenhuizen, North-Holland, Amsterdam, 1979).
[2] P.H. Frampton, S.L. Glashow, and T. Yanagida, Phys.Lett., B548, 119- (2002).

[3] P.F. Harrison, D.H. Perkins, and W.G. Scott, Phys.Lett., B530, 167- (2002).

[4] Review of Particle Properties, Phys. Rev., D54, 1- (1996). 